\title{
Tripotents: a class of strongly clean elements in rings
}

\author{
Grigore Călugăreanu
}

\begin{abstract}
Periodic elements in a ring generate special classes of strongly clean elements. In particular, elements $b$ such that $b=b^{3}$, which are called tripotents and include idempotents, negative of idempotents and order 2 units, are strongly clean. Such elements are determined in $2 \times 2$ matrix rings over commutative domains or over arbitrary division rings and for rings of integers modulo $n$.
\end{abstract}

\section{Introduction}

An element $t$ was called nilpotent in a ring $R$ if $t^{n}=0$ for a positive integer $n$. Among nilpotent elements, the particular case $n=2$ (i.e. zerosquare elements) play a special rôle. Idempotents are also related to $n=2$ so a natural idea is to consider elements $b \in R$ with $b=b^{n}$ for a positive integer $n \geq 3$. But these can be included in a larger subset of the ring: the periodic elements, introduced by $\mathrm{H}$. Bell and studied merely for semigroups by several authors.

An element $a$ in a unital ring $R$ was called clean if there is an idempotent $e$ and a unit $u$ such that $a=e+u$, and strongly clean if $e u=u e$.

We show that periodic elements generate strongly clean elements and in particular, elements $b \in R$ with $b=b^{n}$ for a positive integer $n$ are strongly clean when $n$ is odd. The simplest special case are the elements called tripotents, that is $b=b^{3}$.

Key Words: periodic element, tripotent, strongly clean element, order two unit

2010 Mathematics Subject Classification: Primary 16U99,16U60 Secondary 11Z05,11N69

Received: 13.05 .2017

Revised: 20.06.2017

Accepted:22.06.2017 
It is readily seen that idempotents and negatives of idempotents are tripotents and among units only the order 2 units (also called square roots of 1) are tripotents. Thus, we call a tripotent genuine if it is not an idempotent or a negative of idempotent or an order 2 unit.

We show that $2 \times 2$ matrix rings over commutative (integral) domains or over arbitrary division rings do not possess genuine tripotents.

Idempotents, nilpotents and units are well-known in any $\operatorname{ring} \mathbf{Z}_{n}$ for any positive integer $n \geq 2$. We completely determine the order 2 units and the genuine tripotents for these rings.

We denote by $\operatorname{Per}(R)$ the set of all the periodic elements, $\operatorname{Id}(R)$ the idempotents and $U(R)$ the units of a unital ring $R$. Clearly, $\operatorname{Id}(R) \subset \operatorname{Per}(R)$, $\operatorname{Per}(R) \cap N(R)=0$ and $\operatorname{Per}(R) \cap U(R)=\left\{u \in U(R) \mid u^{m}=1\right\}$.

In mostly all cases we use the simple notation $\bar{a}$ for an integer modulo $n$. However when necessary, we also use the notation $[a]_{n}$.

\section{General results}

Recall that an element $a$ in a ring $R$ was called periodic if $a^{k}=a^{k+m}$ for some positive integers $k, m$. Obviously tripotents are periodic.

The following result will be important.

Proposition 1. Suppose a is a periodic element in a ring and $a^{k}=a^{k+m}$. If $m$ is even then $a^{k}$ is strongly clean and if $m$ is odd, $-a^{k}$ is strongly clean.

Proof. It is well-known (see [1]) that for every periodic element there is a power which is an idempotent. Since we need the details in our proof, suppose $k=q m+r$ is the division with quotient $q$ and reminder $0 \leq r<m$ (with possible $q=0$ if $m \geq k$ ). Observe that $a^{k}=a^{k+m}=a^{k+2 m}=\ldots=a^{k+(q+1) m}$ and $(q+1) m>k$. We just have to multiply $a^{k}=a^{k+(q+1) m}$ with $a^{(q+1) m-k}$ and obtain the idempotent $a^{(q+1) m}$. In particular, if $m \geq k$ (and so $q=0$ ), $a^{m}$ is the required idempotent.

Together with $a^{(q+1) m}, 1-a^{(q+1) m}$ is also (the complementary) idempotent and it is orthogonal to $a^{k}$ (because $a^{k}=a^{k+(q+1) m}$ ).

Next we show that $a^{(q+1) m}=a^{k m}$ if $r \neq 0$. We first rewrite $a^{k}=a^{k+m}$ as $a^{q m+r}=a^{(q+1) m+r}$ and multiply this with $a^{m-r}$. Then $a^{(q+1) m}=a^{(q+2) m}=$ ..., so our claim reduces to $k=q m+r \geq q+1$ since both $m, r \geq 1$.

If $r=0$, that is, $m$ divides $k$ then $a^{k}=a^{q m}=a^{(q+1) m}$ is already idempotent.

Finally, consider $\left[\left(1-a^{(q+1) m}\right)-a^{k}\right]^{m}$. Since the two terms are orthogonal and the left one (the parenthesis) is idempotent, this gives $\left(1-a^{(q+1) m}\right)+a^{k m}$ if $m$ is even and by the claim above, $=1$. Thus $\left(1-a^{(q+1) m}\right)-a^{k}$ is a unit 
and so $a^{k}$ is strongly clean. If $m$ is odd we consider $\left[\left(1-a^{(q+1) m}\right)+a^{k}\right]^{m}$ and similarly we obtain the conclusion $-a^{k}$ is strongly clean.

Remark. As seen above, for a periodic element $a$ with $a^{k}=a^{k+m}, a^{(q+1) m}$ is an idempotent. However, if $r=0$ (recall that $k=q m+r$ ), this is not the smallest power of $a$ which is idempotent: $a^{k}$ is also idempotent.

An important special case, which will be studied in the sequel are the periodic elements $b \in R$ with $k=1$. An element $b=b^{n}$ is called $n$-idempotent (also called potent element in [2]). More specific, for $n=3$, such an element is called a tripotent. We denote by $n \operatorname{Id}(R)$ and $3 \operatorname{Id}(R)$ the corresponding sets of elements. Idempotents (i.e. 2-idempotents) are clearly also $n$-idempotents for any $n \geq 3$. Notice that if $b$ is a tripotent, so is the negative $-b$ and that $3 \operatorname{Id}(R) \cap U(R)=\left\{u \in U(R) \mid u^{2}=1\right\}:=U_{2}(R)$, that is, 1 and order 2 units (also called the 2-torsion subgoup of $U(R)$ ). A tripotent will be called genuine if it is not idempotent $($ so $\neq 0,1)$, not the negative of an idempotent nor an order 2 unit.

If $e$ is a nontrivial idempotent then the negative -e is a tripotent which is not idempotent nor order 2 unit. However, the converse fails: $\overline{4}$ is a genuine tripotent in $\mathbf{Z}_{30}$ which is not the negative of an idempotent.

Since nontrivial $n$-idempotents are zero divisors, integral domains and division rings have only trivial $n$-idempotents.

In particular for tripotents, since $b=b^{3}$ is equivalent to $(b-1) b(b+1)=0$, in any integral domain the only tripotents are $\{-1,0,1\}$.

By Proposition 1, for odd n, n-idempotents are strongly clean and for even $n$, negative of $n$-idempotents are strongly clean. This distinction is natural since the negative of a nonzero idempotent is not idempotent. In particular, tripotents are strongly clean.

From the proof of Proposition 1, we record:

Corollary 2. If $b \in R$ is a tripotent then

(i) $b^{2}$ and $1-b^{2}$ are both idempotents; the converse fails $(2$ is not tripotent in $\mathbf{Z}_{12}$ );

(ii) $b$ and $1-b^{2}$ are orthogonal;

(iii) $1-b-b^{2}$ is an order 2 unit;

(iv) If $b$ is not idempotent then $1+b-b^{2}$ is an order 2 unit.

(v) If $b \in R$ is a tripotent then $R b \oplus R\left(1-b^{2}\right)=R$ is the Pierce decomposition into left (principal) ideals. A symmetric decomposition into right ideals also holds.

(vi) $\operatorname{Id}(R)=3 \operatorname{Id}(R)$ holds for a ring $R$ iff $U_{2}(R)=\{1\}$. 
For commutative rings recall (129.1 [6]) that for every group $G$, there is a (commutative unital) ring whose unit group is isomorphic to $\mathbf{Z}_{2} \times G$. This shows that rings with tripotents which are not idempotents abound. However unit groups without order 2 elements are in this sense exceptional. Indeed (see $129.4[6]$ ): the unit group $U(R)$ of a (commutative unital) ring has trivial 2component iff $R$ is a subdirect sum of domains of characteristic 2.

Example. In $\mathcal{M}_{2}(\mathbf{Z})$, the matrices $\left[\begin{array}{ll}2 & n \\ 0 & 1\end{array}\right]=\left[\begin{array}{ll}1 & n \\ 0 & 0\end{array}\right]+I_{2}$ are strongly clean but not tripotent. However, if $\operatorname{char}(R)=3$ then any strongly clean element whose unit has order 2, is a tripotent.

Indeed, $(e+u)^{3}=e^{3}+u^{3}+3 e u+3 e=e+u$.

Remarks. If all tripotents are central in a ring $R$ then the ring is Abelian and order 2 unit-central, but this is not necessarily. The free $\operatorname{ring} \mathbf{Z}\langle x, y\rangle$ is an example of noncommutative unit-central ring with only trivial idempotents (so Abelian).

Notice that if $2 \in U(R)$ then tripotent-central is equivalent to Abelian (idempotent-central). This follows from a simple representation of tripotent as difference of two idempotents (see [3]), namely: if $b=b^{3}$ then $b=2^{-1}\left(b^{2}+\right.$ b) $-2^{-1}\left(b^{2}-b\right)$.

If all elements are tripotents (rings which satisfy the identity $x^{3}=x$ were investigated by Hirano and Tominaga - see [7]), the ring may not be Boolean (e.g. $\left.\mathbf{F}_{3}\right)$.

It is easy to see that 0 is the only tripotent in the Jacobson radical, 1 and order 2 units are the only (nonzero) tripotents in any local ring and, in any ring with only trivial idempotents, the only nontrivial tripotents (i.e. $\neq 0,1$ ) are order 2 units.

There is a large bibliography on strongly clean matrices the last 10 years (see [5] for a comprehensive survey).

Even for integral $2 \times 2$ matrices, a complete characterization for strongly clean elements is not available yet (but see [4], for extensive results).

It is far more easy to determine the $2 \times 2$ tripotents even over any commutative domain.

Proposition 3. Let $D$ be a commutative (integral) domain. The matrix ring $\mathcal{M}_{2}(D)$ has no genuine tripotents.

Proof. Since $B^{3}=B$ implies $\operatorname{det} B\left(1-\operatorname{det}^{2} B\right)=0$, tripotents have $\operatorname{det} B \in$ $\{0, \pm 1\}$.

If $\operatorname{det} B= \pm 1, B$ is a unit, so it must be an order 2 unit. 
If $\operatorname{det} B=0$, Cayley-Hamilton gives $B^{2}-\operatorname{Tr}(B) B=0_{2}$. Since also $B=$ $B^{3}=\operatorname{Tr}(B) B^{2}=\operatorname{Tr}^{2}(B) B$, we get $B=0_{2}$ or $\operatorname{Tr}^{2}(B)=1$. Hence $B^{2}=B$, that is $B$ is idempotent or $B^{2}=-B$ which are negatives of idempotents.

Remarks. 1) By computation, the tripotent $2 \times 2$ matrices over a commutative domain are:

$$
\begin{aligned}
& I_{2},-I_{2} ;\left[\begin{array}{cc}
a & b \\
c & -a
\end{array}\right] \text { with } a^{2}+b c=1 \text { and } 0_{2},\left[\begin{array}{cc}
a+1 & b \\
c & -a
\end{array}\right] \text { with } a^{2}+a+ \\
& b c=0 \text {, and }\left[\begin{array}{cc}
a-1 & b \\
c & -a
\end{array}\right] \text { with } a^{2}-a+b c=0 .
\end{aligned}
$$

2) The result fails if the domain hypothesis is dropped: in $\mathcal{M}_{2}\left(\mathbf{Z}_{24}\right), \overline{3} I_{2}$ is a genuine tripotent. Actually, if $b$ is any genuine tripotent in a ring $R, b I_{n}$ is a genuine tripotent in $\mathcal{M}_{n}(R)$.

If the commutativity hypothesis is dropped but $D$ is a division ring, we can prove the following

Proposition 4. Let $D$ be any division ring. The matrix ring $\mathcal{M}_{2}(D)$ has no genuine tripotents.

Proof. Since $0_{2}$ is an idempotent, we start with $A=\left[\begin{array}{ll}a & b \\ c & d\end{array}\right] \neq 0_{2}$, matrix with at least one nonzero entry.

We first show that we can always suppose that the $(1,1)$-entry $a \neq 0$.

First observe that if $X$ and $Y$ are similar matrices and $X$ is a tripotent then so is $Y$ (indeed, $\left.X^{3}=X \Longleftrightarrow\left(U X U^{-1}\right)^{3}=U X U^{-1}\right)$. Now, if $d \neq 0$, for $U=\left[\begin{array}{ll}0 & 1 \\ 1 & 0\end{array}\right]=U^{-1}$ we obtain $\left[\begin{array}{ll}0 & 1 \\ 1 & 0\end{array}\right]\left[\begin{array}{ll}a & b \\ c & d\end{array}\right]\left[\begin{array}{ll}0 & 1 \\ 1 & 0\end{array}\right]=\left[\begin{array}{ll}d & c \\ b & a\end{array}\right]$, which has nonzero $(1,1)$-entry. Therefore, if $b \neq 0$ we may suppose $a=d=0$. Now for $V=\left[\begin{array}{ll}1 & 0 \\ 1 & 1\end{array}\right]$ we get $V\left[\begin{array}{ll}0 & b \\ c & 0\end{array}\right] V^{-1}=\left[\begin{array}{cc}-b & b \\ c-b & b\end{array}\right]$ which has nonzero (1,1)-entry. The $c \neq 0$ is similar because $a$ matrix is a tripotent iff its transpose is a tripotent.

Next, for the above matrix $A$, suppose $a \neq 0$. If $U=\left[\begin{array}{cc}1 & 0 \\ -c a^{-1} & 1\end{array}\right]$ then $U A=B=\left[\begin{array}{cc}a & b \\ 0 & d-c a^{-1} b\end{array}\right]$.

(i) If $d-c a^{-1} b \neq 0$ then $B$ is invertible, and so is $A$ (because $U$ is invertible). Hence it is an order 2 unit.

(ii) If $d-c a^{-1} b=0$ then $B=\left[\begin{array}{ll}a & b \\ 0 & 0\end{array}\right]$. Since $A=U^{-1} B$, if $A$ is a tripotent, $\left(U^{-1} B\right)^{3}=U^{-1} B$ and so $\left(B U^{-1}\right)^{3}=B U^{-1}$, that is $B U^{-1}=$ $\left[\begin{array}{ll}a & b \\ 0 & 0\end{array}\right]\left[\begin{array}{cc}1 & 0 \\ c a^{-1} & 1\end{array}\right]=\left[\begin{array}{cc}a+b c a^{-1} & b \\ 0 & 0\end{array}\right]$ is tripotent. 
Further, notice that $\left[\begin{array}{ll}x & y \\ 0 & 0\end{array}\right]^{3}=\left[\begin{array}{cc}x^{3} & x^{2} y \\ 0 & 0\end{array}\right]=\left[\begin{array}{cc}x & y \\ 0 & 0\end{array}\right]$ is equivalent to $x^{3}=x$ and $x^{2} y=y$, and if the matrix is not zero then $x \neq 0$ (indeed, $x=0$ implies $y=0)$. Thus $x^{2}=1$ and so $x \in\{ \pm 1\}$, with arbitrary $y$.

Since $B U^{-1} \neq 0_{2}$ (otherwise $B=0_{2}$ and $A=0_{2}$ ) is a tripotent, $B U^{-1}=$ $\left[\begin{array}{cc} \pm 1 & b \\ 0 & 0\end{array}\right]$ is idempotent if the (1,1)-entry is +1 or is a negative of idempotent if the (1,1)-entry is -1 . Then so is $U^{-1} B=A$, and the proof is complete.

Remark. The property fails for $n \times n$ matrices with $n \geq 3$. Indeed, for any unital ring $R$, in $\mathcal{M}_{3}(R)$ the matrix $B=\left[\begin{array}{lll}0 & 1 & 0 \\ 1 & 0 & 0 \\ 0 & 0 & 0\end{array}\right]$ is a genuine tripotent and the example can be obviously generalized for any $n \geq 3$. Actually, the left-upper $2 \times 2$ corner may be replaced by any order 2 unit.

Finally a useful observation.

Proposition 5. Let $R=R_{1} \times \ldots \times R_{k}$ be a finite direct product of rings and $a=\left(a_{1}, \ldots, a_{k}\right) \in R$. The element $a$ is a tripotent, or idempotent, or order 2 unit, or negative of idempotent iff so are all $a_{i}, 1 \leq i \leq k$, respectively.

Just to simplify the wording in the sequel, let us say that elements in a (finite) subset of a ring are of the same sort if either all are idempotents, or else all are order 2 units, or all are negative of idempotents ("different sort", for denial). Hence

Corollary 6. With above notations, $a$ is a genuine tripotent iff $\left\{a_{1}, \ldots, a_{k}\right\}$ are of different sort.

\section{The tripotents of $\mathbf{Z}_{n}$}

Since local rings are strongly clean and so are direct products, in every $\mathbf{Z}_{n}$, all elements (including tripotents) are (strongly) clean.

In order to determine all the tripotents, we first single out the order 2 units.

The order 2 units.

It is well-known that $\bar{u} \in U\left(\mathbf{Z}_{n}\right)$ iff $(u ; n)=1$ and consequently there are $\varphi(n)$ - the Euler's (totient) function - units in $\mathbf{Z}_{n}$.

More precisely, if $n=p_{1}^{r_{1}} \ldots p_{k}^{r_{k}}$ then $\left|U\left(\mathbf{Z}_{n}\right)\right|=\varphi(n)=n\left(1-\frac{1}{p_{1}}\right) \ldots(1-$ $\left.\frac{1}{p_{k}}\right)=p_{1}^{r_{1}-1}\left(p_{1}-1\right) \ldots p_{k}^{r_{k}-1}\left(p_{k}-1\right)$, that is, $U\left(\mathbf{Z}_{n}\right)$ is a (finite Abelian) group 
of order $\varphi(n)$. Moreover, $U\left(\mathbf{Z}_{n}\right)$ is cyclic iff $n=2,4$, any power of an odd prime or twice any power of an odd prime, is referable to Gauss.

Obviously $-\overline{1}=\overline{n-1}$ is always an order 2 unit in $\mathbf{Z}_{n}$ and $\overline{1} \in U_{2}\left(\mathbf{Z}_{n}\right)$.

Theorem 7. Let $n=p_{1}^{r_{1}} \ldots p_{k}^{r_{k}}$. If $p_{1}=2$ and $r_{1}=1$, then $U_{2}\left(\mathbf{Z}_{n}\right) \cong$ $\mathbf{Z}_{2}^{k-1}=\mathbf{Z}_{2} \times \ldots \times \mathbf{Z}_{2}, k-1$ copies, if $p_{1}=2$ and $r_{1}=2$ or else $p_{1} \geq 3$, then $U_{2}\left(\mathbf{Z}_{n}\right) \cong \mathbf{Z}_{2}^{k}=\mathbf{Z}_{2} \times \ldots \times \mathbf{Z}_{2}, k$ copies, and if $p_{1}=2$ and $r_{1}>2$ then $U_{2}\left(\mathbf{Z}_{n}\right) \cong \mathbf{Z}_{2}^{k+1}=\mathbf{Z}_{2} \times \ldots \times \mathbf{Z}_{2}, k+1$ copies.

Proof. The following is well-known (see [6], chapter XVIII, 128).

For any odd prime $p$, or $p=2, r \leq 2, U\left(\mathbf{Z}_{p^{r}}\right) \cong \mathbf{Z}_{p^{r}-p^{r-1}}$ with $\left|U\left(\mathbf{Z}_{2}\right)\right|=1$, $U\left(\mathbf{Z}_{2^{r}}\right) \cong \mathbf{Z}_{2} \times \mathbf{Z}_{2^{r-2}}$ for any $r>2$ and, for a finite (ring) direct product (sum) $U\left(R_{1} \times \ldots \times R_{k}\right)=U\left(R_{1}\right) \times \ldots \times U\left(R_{k}\right)$. In the case we deal with, $U\left(\mathbf{Z}_{n}\right)=$ $U\left(\mathbf{Z}_{p_{1}^{r_{1}}} \times \ldots \times \mathbf{Z}_{p_{k}^{r_{k}}}\right)=U\left(\mathbf{Z}_{p_{1}^{r_{1}}}\right) \times \ldots \times U\left(\mathbf{Z}_{p_{k}^{r_{k}}}\right) \cong \mathbf{Z}_{p_{1}^{r_{1}-1}\left(p_{1}-1\right)} \times \ldots \times \mathbf{Z}_{p_{k}^{r_{k}-1}\left(p_{k}-1\right)}$ for odd primes or $p_{1}=2, r_{1} \leq 2$, and $U\left(\mathbf{Z}_{n}\right) \cong \mathbf{Z}_{2} \times \mathbf{Z}_{2^{r_{1}-2}} \times \mathbf{Z}_{p_{2}^{r_{2}-1}\left(p_{2}-1\right)} \times$ $\ldots \times \mathbf{Z}_{p_{k}^{r_{k}-1}\left(p_{k}-1\right)}$ if $p_{1}=2, r_{1}>2$.

Since $p_{i}$ and $p_{i}-1$ are coprime, each $\mathbf{Z}_{p_{i}^{r_{i}-1}\left(p_{i}-1\right)} \cong \mathbf{Z}_{p_{i}^{r_{i}-1}} \times \mathbf{Z}_{p_{i}-1}$, so finally

$$
U\left(\mathbf{Z}_{n}\right) \cong \mathbf{Z}_{p_{1}^{r_{1}-1}} \times \ldots \times \mathbf{Z}_{p_{k}^{r_{k}-1}} \times \mathbf{Z}_{p_{1}-1} \times \ldots \times \mathbf{Z}_{p_{k}-1}
$$

in the first case and

$$
U\left(\mathbf{Z}_{n}\right) \cong \mathbf{Z}_{2^{r_{1}-2}} \times \mathbf{Z}_{p_{2}^{r_{2}-1}} \times \ldots \times \mathbf{Z}_{p_{k}^{r_{k}-1}} \times \mathbf{Z}_{2} \times \mathbf{Z}_{p_{2}-1} \times \ldots \times \mathbf{Z}_{p_{k}-1}
$$

in the second case.

Thus, we have already $U\left(\mathbf{Z}_{n}\right)$ decomposed into cyclic groups which give $p_{i}$-components $(i \in\{1, \ldots, k\})$, but also $q$-components with a divisor $q$ of some $p_{i}-1$. Excepting $p_{1}=2$, all the other $p_{i}$ are odd and so $p_{i}-1$ is even.

The elements in $U_{2}\left(\mathbf{Z}_{n}\right)$ form the socle of the 2-component of $U\left(\mathbf{Z}_{n}\right)$, and so is a (finite) elementary 2-group (i.e. a finite direct sum of $\mathbf{Z}_{2}$ ). Since for $p_{i} \geq 3$ each $p_{i}-1$ is even, it will provide an $\mathbf{Z}_{2^{t_{i}}}$ in the decomposition of $\mathbf{Z}_{p_{i}-1}$ and so one $\mathbf{Z}_{2}$ in the decomposition of its socle.

Hence, if $p_{1}=2$ and $r_{1}=1$, then $U_{2}\left(\mathbf{Z}_{n}\right) \cong \mathbf{Z}_{2}^{k-1}=\mathbf{Z}_{2} \times \ldots \times \mathbf{Z}_{2}, k-1$ copies, if $p_{1}=2$ and $r_{1}=2$ or else $p_{1} \geq 3$, then $U_{2}\left(\mathbf{Z}_{n}\right) \cong \mathbf{Z}_{2}^{k}=\mathbf{Z}_{2} \times \ldots \times \mathbf{Z}_{2}$, $k$ copies, and if $p_{1}=2$ and $r_{1}>2$ then $U_{2}\left(\mathbf{Z}_{n}\right) \cong \mathbf{Z}_{2}^{k+1}=\mathbf{Z}_{2} \times \ldots \times \mathbf{Z}_{2}, k+1$ copies.

Using Proposition 5 we can immediately determine the order 2 units

Corollary 8. A unit in $\mathbf{Z}_{n}$ is of order 2 iff each p-component has order at most 2 but at least one component has order 2. For $p>2$ in $\mathbf{Z}_{p^{k}},-\overline{1}$ is the only order 2 unit and in $\mathbf{Z}_{2^{k}}$ there are 3 order 2 units: $-\overline{1}, \overline{2^{k-1}-1}$ and $\overline{2^{k-1}+1}$. 
For the sake of completeness, in what follows we recall some elementary facts on idempotents and order 2 units.

Lemma 9. Let $R$ be a unital ring. Then

(i) If $e \in R$ is an idempotent then $2 e-1 \in U_{2}(R)$. If 4 is not a zero divisor, the converse holds.

(ii) If 2 is invertible and $u \in U_{2}(R)$ then $2^{-1}(u+1)$ is an idempotent.

(iii) Let $R$ be a unital ring with $2 \in U(R)$. The map $f: \operatorname{Id}(R) \longrightarrow U_{2}(R)$ given by $f(e)=2 e-1$ is bijective.

(iv) The function $f(x)=2 x-1$ defined on an unspecified ring $R$ is injective iff 2 is a unit.

(v) Let $e^{\prime}$, e be commuting idempotents. Then $2 e e^{\prime}-e-e^{\prime}+1$ is an idempotent.

(vi) Let $R$ be a ring with commuting idempotents. Then $f(\operatorname{Id}(R))$ is a subgroup of $U_{2}(R)$.

Proof. (vi) Since we deal with order two units (i.e. $\left.u^{-1}=u\right), f(\operatorname{Id}(R))$ is obviously closed to inverses. As for products $f(e) f\left(e^{\prime}\right)=(2 e-1)\left(2 e^{\prime}-1\right)=$ $2\left(2 e e^{\prime}-e-e^{\prime}+1\right)-1=f\left(2 e e^{\prime}-e-e^{\prime}+1\right)$ and we use (i).

Further, in the special case $R=\mathbf{Z}_{n}$ we can add

Corollary 10. (i) If $n$ is any odd positive integer, then $U_{2}\left(\mathbf{Z}_{n}\right)=f(\operatorname{Id}(R))=$ $2 \operatorname{Id}(R)-1$. The number of order 2 units is equal to the number of idempotents, and this is $2^{k}$ if $n$ has exactly $k$ (distinct) prime divisors.

(ii) $f$ is injective on $\operatorname{Id}\left(\mathbf{Z}_{n}\right)$ for any $n$ divisible by 4 .

(iii) For $n=2^{s} m$ with odd $m$ and $s>2, \frac{\bar{n}-1}{2}$ is an order 2 unit in $\mathbf{Z}_{n}$ which is not image of idempotent through $f$.

Proof. (i) Let $e, e^{\prime}$ be idempotents in $\mathbf{Z}_{n}$ for $n=2^{s} m$ with odd $m$ and $s \geq 2$. Suppose $f\left(e^{\prime}\right)=f(e)$ that is $2\left(e^{\prime}-e\right)=0$. Since $0 \leq e^{\prime}-e \leq n-1$, if $e^{\prime} \neq e$, this means $e^{\prime}-e=\frac{n}{2}=2^{s-1} m$ and so $e+2^{s-1} m$ is an idempotent. Hence $\left(e+2^{s-1} m\right)^{2}=e+2^{s-1} m$ and so $2 e=1-2^{s-1} m$, a contradiction in a ring of integers modulo even number. Therefore $e^{\prime}=e$.

(iii) Indeed, $\left(2^{s-1} m-1\right)^{2}=2^{s} m\left(2^{s-2} m-1\right)+1 \equiv 1(\bmod n)$ and $2^{s-1} m-$ $1=f\left(2^{s-2} m\right)$, with nilpotent $2^{s-2} m$.

With this at hand we can prove the following

Proposition 11. For every even $n$, the order 2 units are obtained as images of idempotents through $f$ with only one exception: if 8 divides $n$. In this case $f\left(\operatorname{Id}\left(\mathbf{Z}_{n}\right)\right.$ is an index 2 subgroup of $U_{2}\left(\mathbf{Z}_{n}\right)$ and $U_{2}\left(\mathbf{Z}_{n}\right)-f\left(\operatorname{Id}\left(\mathbf{Z}_{n}\right)=\right.$ 
$\overline{\frac{n}{2}-1} \cdot f\left(\operatorname{Id}\left(\mathbf{Z}_{n}\right)\right.$, that is, the order 2 units which are not images of idempotents form a coset modulo $\frac{\bar{n}-1}{2}$.

Proof. Suppose $n=2^{s} p_{2}^{r_{2}} \ldots p_{k}^{r_{k}}$ for odd primes $p_{i}(i \in\{2, \ldots, k\})$. Again from Theorem 7, we already know that $U_{2}\left(\mathbf{Z}_{n}\right) \cong \mathbf{Z}_{2}^{k+1}$ if $s>2, U_{2}\left(\mathbf{Z}_{n}\right) \cong \mathbf{Z}_{2}^{k}$ if $s=2$ and $U_{2}\left(\mathbf{Z}_{n}\right) \cong \mathbf{Z}_{2}^{k-1}$ is $s=1$. Since the number of idempotents is $2^{k}$ in all cases, the order 2 units are obtained from idempotents using the map $f$ given above, bijective in the second case and just surjective in the third.

So order 2 units are always obtained from idempotents through $f$, excepting the case $s>2$. In this case $f$ provides only half of the order 2 units (by Lemma 10, $\left|f\left(\operatorname{Id}\left(\mathbf{Z}_{n}\right)\right)\right|=2^{k}$ ) and, according to Lemma 9, this subset is an index 2 subgroup of $U_{2}\left(\mathbf{Z}_{n}\right)$. By Lemma 9 (iii) $\frac{\bar{n}}{2}-1$ is an order 2 unit which is not in $f\left(\operatorname{Id}\left(\mathbf{Z}_{n}\right)\right)$, so the remaining order 2 units are in the coset $\overline{\frac{n}{2}-1} \cdot f\left(\operatorname{Id}\left(\mathbf{Z}_{n}\right)\right.$.

Examples. 1) $\operatorname{Id}\left(\mathbf{Z}_{30}\right)=\{\overline{0}, \overline{1}, \overline{6}, \overline{10}, \overline{15}, \overline{16}, \overline{21}, \overline{25}\}$. These are mapped onto $U_{2}\left(\mathbf{Z}_{30}\right)=\{\overline{1}, \overline{11}, \overline{19}, \overline{29}\}: f(\overline{0})=f(\overline{15})=\overline{29}, f(\overline{1})=f(\overline{16})=\overline{1}, f(\overline{6})=$ $f(\overline{21})=\overline{11}$ and $f(\overline{10})=f(\overline{25})=\overline{19}$.

2) For $n=24$, the idempotents are $\operatorname{Id}\left(\mathbf{Z}_{24}\right)=\{\overline{0}, \overline{1}, \overline{9}, \overline{16}\}$. These are mapped by $f$ into $\{\overline{23}, \overline{1}, \overline{17}, \overline{7}\}$ but there are another four order 2 units, namely $\{\overline{5}, \overline{11}, \overline{13}, \overline{19}\}$. As noticed in Lemma 9 , since $\overline{7} \cdot \overline{17}=\overline{23}, \overline{7} \cdot \overline{23}=\overline{17}, \overline{17} \cdot \overline{23}=\overline{7}$, $\{\overline{1}, \overline{7}, \overline{17}, \overline{23}\}$ is an index 2 subgroup of $U_{2}\left(\mathbf{Z}_{24}\right)$ and $\{\overline{5}, \overline{11}, \overline{13}, \overline{19}\}$ are not images of idempotents through $f$. However this a coset $\overline{11} \cdot f\left(\operatorname{Id}\left(\mathbf{Z}_{24}\right)\right)=$ $\overline{11} \cdot\{\overline{1}, \overline{7}, \overline{17}, \overline{23}\}=\{\overline{5}, \overline{11}, \overline{13}, \overline{19}\}\left(\overline{\frac{n}{2}-1}=\overline{11}\right.$ in Lemma 9 (iii) $)$.

The genuine tripotents.

As observed in Corollary $6,[x]_{n}$ is a genuine tripotent iff all $[x]_{p_{1}^{r_{1}}}, \ldots,[x]_{p_{k}^{r_{k}}}$ are (tripotents but) of different sort.

First we prove the following

Proposition 12. If $n=p^{s} q^{r}$ with different odd primes $p, q$ and $s, r \geq 0$ then $\mathbf{Z}_{n}$ has no genuine tripotents.

Proof. Suppose $[x]_{n}$ is a tripotent in $\mathbf{Z}_{n}$. Then $[x]_{p^{s}}=\bar{x}$ is a tripotent in $\mathbf{Z}_{p^{s}}$ and $[x]_{q^{r}}=\widehat{x}$ is a tripotent in $\mathbf{Z}_{q^{r}}$. As already noticed, these can be only $\overline{0}, \overline{1}$ or $-\overline{1}$. Excepting $(\overline{0}, \widehat{0}),(\overline{1}, \widehat{1})$ and $(-\overline{1},-\widehat{1})$ which are clearly of the same sort, we have three other possibilities (and symmetric): $(\overline{0}, \widehat{1})$ both idempotents, $(-\overline{1}, \widehat{1})$ both in $U_{2}\left(\mathbf{Z}_{n}\right)$ and $(\overline{0},-\widehat{1})$ both negatives of idempotents. Therefore, 
in all possible cases $[x]_{p^{s}}$ and $[x]_{q^{r}}$ are of the same sort, that is, there are no genuine tripotents.

Remarks. (i) Since $\operatorname{gcd}\left(p^{s} ; q^{r}\right)=1$, there are integers $c, d$ such that $c p^{s}+d q^{r}=1$. Then $c p^{s}$ and $d q^{r}$ are the only (two) nontrivial (complementary) idempotents. For $\overline{0}$ and $\overline{1}$, the negatives give $\overline{0}$ and $-\overline{1}$, the last known as order 2 unit.

Thus, $-c p^{s}$ and $-d q^{r}$ are the only (two) nontrivial negatives of idempotents.

(ii) This fails for three or more primes. For $n=3 \cdot 5 \cdot 7=105$ take $[6]_{n}$. Then $\phi\left([6]_{n}\right)=\left([6]_{3},[6]_{5},[6]_{7}\right)=\left([0]_{3},[1]_{5},-[1]_{7}\right)$, components which are of different sort and $[6]_{105}$ is a genuine tripotent.

The only case left is $p_{1}=2$. By the above discussion, an element $[x]_{n} \in \mathbf{Z}_{n}$ for $n=2^{s} p_{2}^{r_{2}} \ldots p_{k}^{r_{k}}$ is a genuine tripotent iff $[x]_{2^{s}},[x]_{p_{2}^{r_{2}}}, \ldots,[x]_{p_{k}}^{r_{k}}$ are of different sort.

Since $\mathbf{Z}_{2^{s}}$ are local, the odd classes are units and the even classes are nilpotents. Such rings have only trivial idempotents. Therefore, the situation is similar to the odd case, but excepting $\overline{0}, \overline{1},-\overline{1}$ we may have extra order 2 units. Not for $\mathbf{Z}_{2}$ or $\mathbf{Z}_{4}$ but for all $\mathbf{Z}_{2^{s}}$ with $s \geq 3$.

Hence we can state the following

Proposition 13. Let $n=2^{s} p_{2}^{r_{2}} \ldots p_{k}^{r_{k}}$ and $[x]_{n} \in \mathbf{Z}_{n}$.

(i) If $k=2$ and $s \leq 2, \mathbf{Z}_{n}$ has no genuine tripotents.

(ii) If $k=2$ and $s \geq 3, \mathbf{Z}_{n}$ has genuine tripotents. These are listed depending on the reminder of the division of $p_{2}^{r_{2}}$ to $2^{s}$.

(iii) If $k>2, \mathbf{Z}_{n}$ has genuine tripotents.

Proof. (i) Exactly like the odd case (Proposition 12).

(ii) The genuine tripotents are exactly the classes $[x]_{n}$ such that $[x]_{2^{s}} \in$ $\left\{\overline{3}, \overline{5}, \ldots, \overline{2^{s}-3}\right\}$ and $[x]_{p_{2}}^{r_{2}}=\overline{0}$, because only such components are of different sort. Such tripotents do exist: suppose $p_{2}^{r_{2}}=2^{s} c+d$ is the division with quotient $c$ and reminder $d$. If $d \neq 1,2^{s}-1$ then $p_{2}^{r_{2}}$ is a genuine tripotent, and if $d \in\left\{1,2^{s}-1\right\}$ then $3 p_{2}^{r_{2}}$ is a genuine tripotent.

More precisely, we have to check the reminders modulo $2^{s}$ of the multiples of $p_{2}^{r_{2}}$, which are less then $n$ (i.e. $2^{s}-1$ such multiples).

If $d \neq 1,2^{s}-1$, then $\overline{p_{2}^{r_{2}}}$ and all odd multiples $k \overline{p_{2}^{r_{2}}}$ with $k d \in\left\{3,5, \ldots, 2^{s}-3\right\}$ are genuine tripotents (and the number of these is the integer part $\left[\frac{2^{s}-3}{d}\right]$ ). If $d \in\left\{1,2^{s}-1\right\}$ then $\overline{p_{2}^{r_{2}}} \in U_{2}\left(\mathbf{Z}_{n}\right)$, so is not genuine. Now the odd multiples 
$k \overline{p_{2}^{r_{2}}}$ such that $k \in\left\{3,5, \ldots, 2^{s}-3\right\}$ are all the genuine tripotents (and these are exactly $2^{s-1}-2$ ).

(iii) Now we can vary the 2-component as in (ii) but also the other (at least two) $p_{i}$-components in $\{\overline{0}, \overline{1},-\overline{1}\}$ in order to have components of different sort. A similar discussion shows that such tripotents do exist and how these can be listed.

Examples. 1) In $\mathbf{Z}_{24}$ take $\overline{3}$ or $\overline{21}$. For both $[3]_{3}=[21]_{3}=\overline{0}$ and $[3]_{8}=\overline{3}$ respectively, $[21]_{8}=\overline{5}$. These are the only two genuine tripotents.

2) In $\mathbf{Z}_{56}$ we have only $\overline{21}$ and $\overline{35}$ with the 7 -component $\overline{0}$ (see (ii) in the previous proof).

3) In $\mathbf{Z}_{30}$ take $\overline{4}$ : now $\phi(\overline{4})=\left([0]_{2},[1]_{3},-[1]_{5}\right)$ so this is a tripotent and a genuine one (components of different sort). There is only one other possibility: $\left([0]_{2},-[1]_{3},[1]_{5}\right)$, that is $\overline{26}=-\overline{4}$.

More general, one can check that for any odd positive integer $p, \overline{p+1}$ is a genuine tripotent in $\mathbf{Z}_{2 p(p+2)}$ and so is the negative $\overline{2 p^{2}+3 p-1}$.

If $p$ is a prime, these are the only genuine tripotents: there are no possible components of different sort if a tripotent is odd, so the 2-component must be $\overline{0}$ (i.e. the tripotent is even). There are only two possibilities: $p$-component $\overline{1}$ and $p+2$-compotent $-\overline{1}$, which gives $\overline{p+1}$ or vice-versa, with its negative $\overline{2 p^{2}+3 p-1}$.

\section{References}

[1] H. E. Bell A commutativity study for periodic rings. Pacific J. of Math. 70 (1) (1977), 29-36.

[2] H. E. Bell, A. Yaqub On generalized periodic-like rings. International J. of Math. and Math. Sciences. Volume 2007, 5 pages.

[3] A.M. Bikchentaev, R.S. Yakushev Representation of tripotents and representations via tripotents. Linear Algebra and its Applications 435 (2011) 2156-2165.

[4] H. Chen On $2 \times 2$ strongly clean matrices. Bull. Korean Math. Soc. 50 (1) (2013), 125-134.

[5] A. J. Diesl, T. J. Dorsey Strongly clean matrices over arbitrary rings. Journal of Algebra 399 (2014), 854-869.

[6] L. Fuchs Infinite Abelian Groups. Vol. 2, Academic Press 1973. 
[7] Y. Hirano and H. Tominaga Rings in which every element is the sum of two idempotents. Bull. Austral. Math. Soc. 37 (2) (1988), 161-164.

[8] D. Khurana, G. Marks, A. K. Srivastava On unit-central rings. Trends in Math., Springer, Advances in Ring Theory, 205-212.

[9] T. Y. Lam A first course in noncommutative rings. Second Edition, GMT 131, Springer Verlag, 2001.

Grigore Călugăreanu,

Department of Mathematics,

Babes - Bolyai University,

str. Kogălniceanu 1, Constanta, Romania.

Email: calu@math.ubbcluj.ro 